\title{
DISCLAIMER
}

This report was prepared as an account of work sponsored by an agency of the United States Government. Neither the United States Government nor any agency thereof, nor any of their employees, makes any warranty, express or implied, or assumes any legal liability or responsibility for the accuracy, completeness, or usefulness of any information, apparatus, product, or process disclosed, or represents that its use would not infringe privately owned rights. Reference herein to any specific commercial product, process, or service by trade name, trademark, manufacturer, or otherwise does not necessarily constitute or imply its endorsement, recommendation, or favoring by the United States Government or any agency thereof. The views and opinions of authors expressed herein do not necessarily state or reflect those of the United States Government or any agency thereof.

\section{ANALYSIS OF COMPLEX SYSTEMS USING NEURAL NETWORKS}

\section{Robert E. Uhrig}

Department of Nuclear Engineering, University of Tennessee, Knoxville, TN 37996-2300 and

Instrument and Control. Division, Oak Ridge National Laboratory, Oak Ridge, TN 37831-6005

To be presented before and included in the

Proceedings of The Second International Forum on

\section{EXPERT SYSTEMS AND COMPUTER SIMULATION IN ENERGY SYSTEMS}

\author{
Erlangen, Germany
}

March 17-20, 1992 


\title{
ANALYSIS OF COMPLEX SYSTEMS USING NEURAL NET,VORKS
}

\author{
Robert E. Uhrig
}

Department of Nuclear Engincering, University of Tennessee, Knoxville, TN 37996.2300 and

Instrumentation and Control Division, Oak Ridge National Laboratory, Oak Ridge. TN 37831.6005

\section{ABSTRACT}

The application of neural networks, alone or in conjunction with other advanced technologies (expert systems, fuzzy logic, and/or genetic algorithms), to some of the problems of complex engineering systems has the potential to enhance the safety, reliability, and operability of these systems. Typically, the measured variables from the systems are analog variables that must be sampled and normalized to expected peak values before they are introduced into neural networks. Often data must be processed to put it into a form more acceptable to the neural network (e.g., a fast Fourier transformation of the time-series data to produce a spectral plot of the data). Specific applications described include: 1) Diagnostics: State of the Plant, 2) Hybrid System for Transient Identification, 3) Sensor Validation, 4) Plant-Wide Monitoring, 5) Monitoring of Performance and Efficiency, and 6) Analysis of Vibrations. Although the specific examples described deal with nuclear power plants or their subsystems, the techniques described can be applied to a wide variety of complex engineering systems.

\section{INTRODUCTION}

The application of neural networks, a rapidly evolving technology used extensively in defense applications, alone or in conjunction with other advanced technologies (expert systems, fuzzy logic, and/or genetic algorithms), to some of the problems of complex engineering systems has the potential to enhance the safety, reliability and operability of these systems.

Neural networks have emerged during the past decade from an obscure field that had been discredited by perceived inadequacies into one of the fastest growing technologies in information processing. In the past three years, several books have been written that present the theory and general application of neural networks in a lucid manner. [e.g., Hecht-Nielson 1990 and Simpson 1990]. Hence, the discussion in this article will be limited to describing the general nature and characteristics of neural networks and their potential application to a wide variety of engineering applications. Specific applications to the nuclear power field will be cited because of the background of the author, but the applications envisioned in this article are to a wide variety of complex systems (e.g., space systems, chemical plants, production lines, etc.).

\section{NEURAL NETWORKS}

A network of artificial neurons, usually called an artificial neural network (ANN), is a data processing system consisting of a number of simple, highly interconnected processing elements in an architecture inspired by the structure of the cerebral cortex portion of the brain. Hence, neural networks are often capable of doing things which humans or animals do well but which conventional computers often do poorly. Neural networks exhibit characteristics and capabilities not provided by any other technology. Neural networks have the ability to respond in real-time to the changing system state descriptions provided by continuous sensor inputs. Neural networks may be designed so as to classify an input pattern as one of many predefined types (e.g., the various fault or transient states of an engineering system) or to create, as needed, categories or classes of system states which can be interpreted by a human operator. Once a neural network has been trained to recognize the -various conditions or states of a complex system, it only takes one recall cycle of the neural network, typically a few milliseconds, to detect a specific condition or state.

\section{APPLICATIONS OF NEURAL NETWORKS}

The work described here deals with complex systems, often large complex systems, or parts of such systems that can be isolated from the total system. Typically, the measured variables from the systems are analog variables that must be sampled and normalized to expected peak values before they are introduced into neural networks. The neural networks are usually simulated on modern high-speed digital computers that carry out the calculations serially. However, it is possible to implement neural networks using specially designed microchips where the network calculations are carried out in parallel, thereby providing virtually instantaneous outpu's (microsecond response times) for each set of inputs. Speed-ups of 100 to 1000 over simulation systems are possible using appropriate microchips to implement the neural network. 
would be a quantity (usually a binary variable) jdentifying the specific transient. Several variations of each transient may be needed in the training sets, if the pattern relationships change with the severity of the transient. In the training, it is important to cover the entire dynamic range of the variables that may occur during the monitoring period. The trained neural network can be made more robust against variations in the input data by introducing a small amount (typically 5 to 10\%) of noise into the input variables during the training.

Often, the nature of a complex system is such that it is not possible to put it through the transients nece: sary to provide the data for training. Under these circumstances, it may be necessary to simulate the system on a computer (or utilize a full-fidelity training simulator if one is available) to produce the time records of variables during transients needed to train the neural network. The training procedure described above is used except that the outputs for training are from the computer simulation or simulator.

Exnmple. Experience to date using data from the modem (1989 installation) Singer-Link training simulator at TVA's Watts Bar Nuclear Power Plant to identify seven transients and steady state operation has been very successful, even with the inclusion of noise in the simulator signals. (Bartlett 1990) Some 27 variables (reduced from 80 by combining data from the four loops into an equivalent single average loop) were used to iden'ify seven different transients (loss of coolant in the hot le ${ }_{3}$ of the reactor coolant system (RCS), loss of coolant in the cold leg of the RCS, main steam-line break in containment, main feedwater line break in containment, total loss of off-site power, control rod ejection, and steam generator tube leak). Time records for the 27 variables constituted the input vectors to the neural network for training. In all cases, the network was able to immediately identify the transients. Even in the presence of $5 \%$ noise, the network was able to identify the transient before the automatic shutdown system shut down the plant. (Guo 1992)

This work is being extended by using genetic algorithms and sensitivity analysis to identify the most important variables for each input. (Guo 1992) Then individual neural networks for each transient, using the most important variables, are being designed for each transient and trained to identify variations of each transient. Since the optimization process provides an index of the importance of each input, weighting of the inputs with these coefficient further improves the ability of the neural network to identify the transients.

\section{HYBRID SYSTEM FOR TRANSIENT IDENTIFICATION}

A unique hybrid system has been developed at the University of Tennessee (Tsoukalas, Ikonomopoulos, and Uhrig, 1991) for the identification of transients in complex systems. It couples a rule-based expert system using fuzzy 
logic to a pretrained artificial neural network and uses a model-referenced approach to help in the identification of noisy data. The expert system performs the basic interpretation and processing of the model data. A set of pre-trained artificial neural networks provide the models of the transients. Membership functions (from fuzzy logic) that condense information about a transient in a form convenient for a rule-based identification system and characterize the transients are the outputs of the neural networks. After training, the system is capable of performing faster than real-time.

Example. To demonstrate the use of this system, the same eight transients from the Watts Bar Nuclear Power Plant Simulator discussed above were used as inputs to the neural network. Time series of these the variables during the transient were the inputs to the neural network. The output is a membership function that contains only two pieces of information about the input transient, the peak value and the time at which this peak occurs. Tests show the system is capable of differentiating between the these transients, even when the inputs are corrupted with up to $20 \%$ random noise.

\section{SENSOR VALIDATION}

The usefulness of information provided to ope:ating personnel is dependent upon the validity of the signals coming from sensors as well as the ability to convert such data into meaningful information, presenting it in an understandable format, and providing an analysis of the current situation. A neural network paradigm has been developed at the University of Tennessee [Upadhyaya, Eryurek, and Mathai] for automated sensor validation during both steady-state and transient operations. The use of neural networks for signal estimation has the advantage that the functional form relating a set of process variables is defined by the neural network system (model of the process) and is implicitly nonlinear. Once the network is properly trained, future predictions can be made in real time. Furthermore, the state estimation using a neural network is less sensitive to measurement noise compared to direct model-based techniques.

For signal validation, a neural network model of the process (or a portion of the process) can be developed using experimental data. The input layer of artificial neurons correspond to several input signals, and the output layer usually has just one signal which is to be predicted or estimated. The neural network is trained over the range of anticipated operating conditions while the system is known to be operating properly. Then the neural network model repeatedly predicts the output value, based on the actual inputs. As a sensor deteriorates or fails, the predicted output value (based on the input values and the neural network model) deviates from the measured value (which is erroneous). A comparison of the predicted and actual values will indicate the validity of the sensor reading.
Example. Tests with data from the Experimental Breeder Reactor-II (EBR-II), a liquid metal fast breeder reactor at the Idaho National Engineering Laboratory and a commercial four-loop PWR power plant have shown that this technique can be used to validate data from sensors placed in nuclear power plants [Upadhyaya, Eryurek, and Ma:hai]. Networks for the validation of readings of hot leg temperature, reactor power and control rod position for a 4loop PWR demonstrated the validity of this approach. For the hot leg temperature reading, the input signals were reactor power, pressurizer level and cold leg temperature. The network had 22 nodes in the hidden layer and required 300 iterations for 150 input patterns using adaptive thresholding to accele rate the backpropagation training. The standard deviation achieved was $0.19^{\circ} \mathrm{C}$.

\section{PLANT.WIDE MONITORING USING NEURAL NETWORKS}

The technique for sensor validation has been expanded into a plant-wide monitoring system [Upadhyaya, Eryurek, and Mathai] by using an autoassociative neural network where the inputs and the outputs are identically the same variables. The number of artificial neurons in the intermediate layer (usually about three times the number of input nodes) is selected to minimize the training time and maximize the ability of the neural network to generalize. The network is trained over the range of operation using the same data for the input vector and the desired output vector. Backpropagation using a sigmoidal function with an adjustable coefficient was used to train the network. The network is trained when the system is operating properly. Under these conditions, its outputs represent estimates of the instantaneous values of the output variables, and all of these estimates are virtually identical to the actual outputs. When a sensor begins to drift or an error is introduced into a data channel, the predicted value and the actual value differ. Hence, monitoring the differences between the estimates predicted by the neural network and the actual values from the system provides a method of identifying drift or instrumentation system failure. An alternative interpretation of these differences might be that the input-output relationship of the system from which the signals come may be changing due to system failure or changes of some sort.

Example \#1. This technique was applied to data from eighteen signals from the EBR-II during ascension in power from $45 \%$ to $100 \%$. To reduce the training required, two separate networks were used, one for eight variables related to the primary system and the other for the ten variables related to the secondary system and balance of plant. Errors between estimates predicted by the neural network and the actual values were usually less than $0.5 \%$. Further reduction of this difference should be possible by removing the mean values of the signals prior to introducing ther into the neural network. When one of the sensors failed or an error 
was introduced into one of the sensor outputs (the inputs to the network), the corresponding output of the network changed only slightly. Hence, a difference between the network output (the predicted value of the variable) and the actual variable identifies that variable as the one with a problem.

Example \#2. Check valve failures at San Onofre Unit 1 on November 21, 1985 led to the NRC (Nuclear Regulatory Commission) issuing NUREG-1190 that discussed the reasons for the failures of the five check valves and how the check valve failures induced water hammers to the feedwater system. Although there are many possible failure mechanisms for check valves, the most common problems associated with check valve failures are due to system flow oscillations or system piping vibrations. These vibrations and oscillations induce check valve component wear and thus component failure. Many of them emit sounds that can be detected by monitoring acoustical emissions from the valve body.

A technique involving the use of a neural network for the analysis of acoustical data from check valves has been developed at the University of Tennessee under support of the Electric Power Research Institute (EPRI). [Iconomopoulos and Uhrig] It involves training a neural network to model the behavior of the check valve. In this case, the power spectral density (PSD) of the sampled timeseries at one point on the check valve is used as the input to the neural network and the PSD of the sampled time-series at another point is the desired output of the neural network. The network is trained while the flow varies over the normal range of operation when the valve is known to be operating properly. The neural network is then used in a monitoring mode to predict the output sensor PSD from the input PSD and a comparison is made between the predicted and actual output PSDs. Deviations indicate that the inter-relationship between the input and output signals has changed due to a change (failure) of the valve.

\section{MONITORING OF PERFORMANCE AND EFFICIENCY}

Of the hundreds of values of quantities used to calculate indices of performance (efficiency, specific fuel/energy consumption, etc.), of complex systems, many remain constant because they are related to the design of the system. With neural networks, it is possible to model the dynamic portion of the process using only those variables that change during operation, provided that the other quantities do not change. Once the neural network model has been developed, it is then possible to determine the sensitivity of each output (efficiency or performance index) to changes in each input. The largest sensitivity coefficients indicate the input variables that most influence efficiency and performance. Hence, efforts to improve performance should concentrate on these quantities.
Example. This technique was applied to TVA's Sequoyah Unit \#1 Nuclear Power plant. Of approximately 130 quantities used to calculate the heat rate (inversely related to efficiency), only 26 actually change with time. Records of these 26 variables for about 45 weekly measurements were provided by TVA personnel to the University of Tennessee where they were used as inputs to a neural network to develop a model of the thermodynamic processes of the plant [Guo and Uhrig, 1992]. The training of the network involved the use of a Kuhonen neural network technique to cluster the data into 22 clusters with an Euclitean distance of about 1.0. Then the centroids of these clusters were used as inputs to a neural network. Backpropagation was used for training until the overall system error was about $0.01 \%$. When the original data (not the centroids of the clusters used in training) were introduced into this model for recall, the results were usually within $0.1 \%$ of the measured values. When the same thermodynamic data from Unit-2 (a very similar, but not identical, unit) was put into the Unit \#1 network for recall, the output heat rates usually agreed within $0.5 \%$ of the measured values. This indicates that the neural network models of the thermodynamics of these two nuclear power plants are very similar.

The neural network model was then used for a sensitivity analysis in which the partial derivative of each ontpu? variable with respect to each individual input value was evaluated using values from the neural network model. The larger the partial derivative, the more influence the particular input variable has on the output (efficiency or heat rate). The sensitivity of the heat rate with respect to different variables can give guidance to the utility as to which efforts to improve efficiency are likely to be most effective. In some cases it may not be possible to change an input variable, even though it may be very important to efficiency (e.g., it is virtually impossible to control the river or ocean temperature). It is also useful to compare the importance of a particular input variable with the cost of controlling it.

\section{ANALYSIS OF VIBRATIONS}

The flow of fluids (water, air or steam) as well as rotating machinery can induce vibration and shock in most complex systems. Vibration per se is not necessarily bad it its amplitudes and the associated forces are within acceptable limits. Indeed, vibration in machinery can be the source of much information about the various systems involved. Analysis of vibration data can identify the operational problems of rotating machinery. Such analyses have proven very effective in identifying potential failures before they cecur. Indeed, it is sometimes possible to estimate the remaining life of certain systems, once a fault has been detected. Hence, there is considerable motivation to design instrumentation to automatically perform this analysis on a real-time basis in a reproducible manner. 
Because it is difficult for neural networks to de al with power spectral densities that have narrow peaks (which are typical of PSDs taken on rotating machinery) the PSDs were transformed using a recirculation neural network to reduce their dimensionality. This effectively spread the information contained in the peaks over the whole frequency spectrum and put the data in a form more appropriate for analysis using backpropagation training and recall. The result was that the accuracy in fault detection was very high (97 to $100 \%)$.

Example 1. The data available for analysis consisted of spectral density measurements at nine different positions on each of 246 "laminar flow" table rollers in a steel sheet manufacturing mill. This project involves the application of neural network technology to the interpretation and the automation of the processes that typically require expertise to interpret the spectrum measurements. The problems to be identified were misalignment, improper lubrication, wear in motor bushes to inboard and outboard bearings, and looseness as well as combinations of these faults. Each of the nine networks, one for each sensor position, was trained to identify each of these problems using data from data taken on faulty systems. Then data from machines not used in the training were presented to the trained neural networks to identify the faults. When all nine networks gave the same result, it was clear that there was one overriding problem. When networks gave different results, there were usually more than one problem. A less severe problem in a bearing near a sensor could override the influence of a more severe problem located further away.

Example 2. A program for the :analysis of vibration data from ball bearings has been carried out at the University of Tennessee [Alguindigue and Uhrig, 1991] under the sponsorship of Electricite de France, the French national utility). This project involved both training the neural networks to recognize predefined conditions and using the networks for data clustering based on the inherent statistical features of the data. Of 71 data patterns, 17 were chosen for training the network. Seven patterns had no labels (identified faults) were collected while the bearing was deteriorating and may represent more than one fault condition. Of the remaining 64 patterns, 62 were classified correctly, a $97 \%$ success rate. Two normal patterns were classified as faulty, indicating a conservative classification.
CONCLUSION

In the light of the experience described above, it is clear that neural networks offer an interesting, challenging, and productive means of addressing many of the problems that occur in complex systems. Although much of the work described has been to demonstrate feasibility of the specific approach, the results are encouraging and indicate that the neural network techniques have the potential to enhance the performance and safety of complex systems in a cost effective way.

\section{REFERENCES}

ALGUINDIGUE I., \& UHRIG R.E.: 1991, "Vibration Monitoring with Artificial Neural Networks," Proceedings of the SMORN VI Symposium on Nuclear Reactor Surveillance and Diagnostics, Gatlinburg, TN, May 19-24.

BARTLETT E. E.: 1990, "Nuclear Power Plant Status Diagnostics Using Simulated Condensation: An AutoAdaptive Computer Learning Technique," Unpublished Ph.D. Dissertation, University of Tennessee, Knoxville, TN.

GUO Z.: 1992, "Nuclear Power Plant Fault Diagnostics and Thermal Performance Studies using Neural Networks," Ph.D. dissertation, University of Tennessee.

GUO Z., \& UHRIG R. E.: 1992, "Use of Artificial Neural Networks to Analyze Nuclear power Plant Performance" Nuclear Technology (In Press).

HECHT-NIELSEN R.: 1989, Neurocomputing, Addison Wesley Publishing Co., Reading, MA.

IKONOMOPOULOS A, \& UHRIG R.E.: 1992, private communication.

SIMPSON P.: 1990, Artificial Neural Systems, Pergamon Publishing Co., New York.

TSOUKALAS L, \& IKONOMOPOULOS A, Uhrig R.E.: 1991, "Hybrid Expert System--Neural Network Methodology for Transient Identification," Proceedings of the American Power Conference, (In press) Chicago, IL, April 29-May 1.

UPADHYAYA B.R., \& MATHAI G., ERYUREK E.: 1990, "Development and Application of Neural Network Algorithms for Process Diagnostics," Proceedings of the 29th IEEE Conference on Decision and Control, Honolulu, Hawaii, December. 


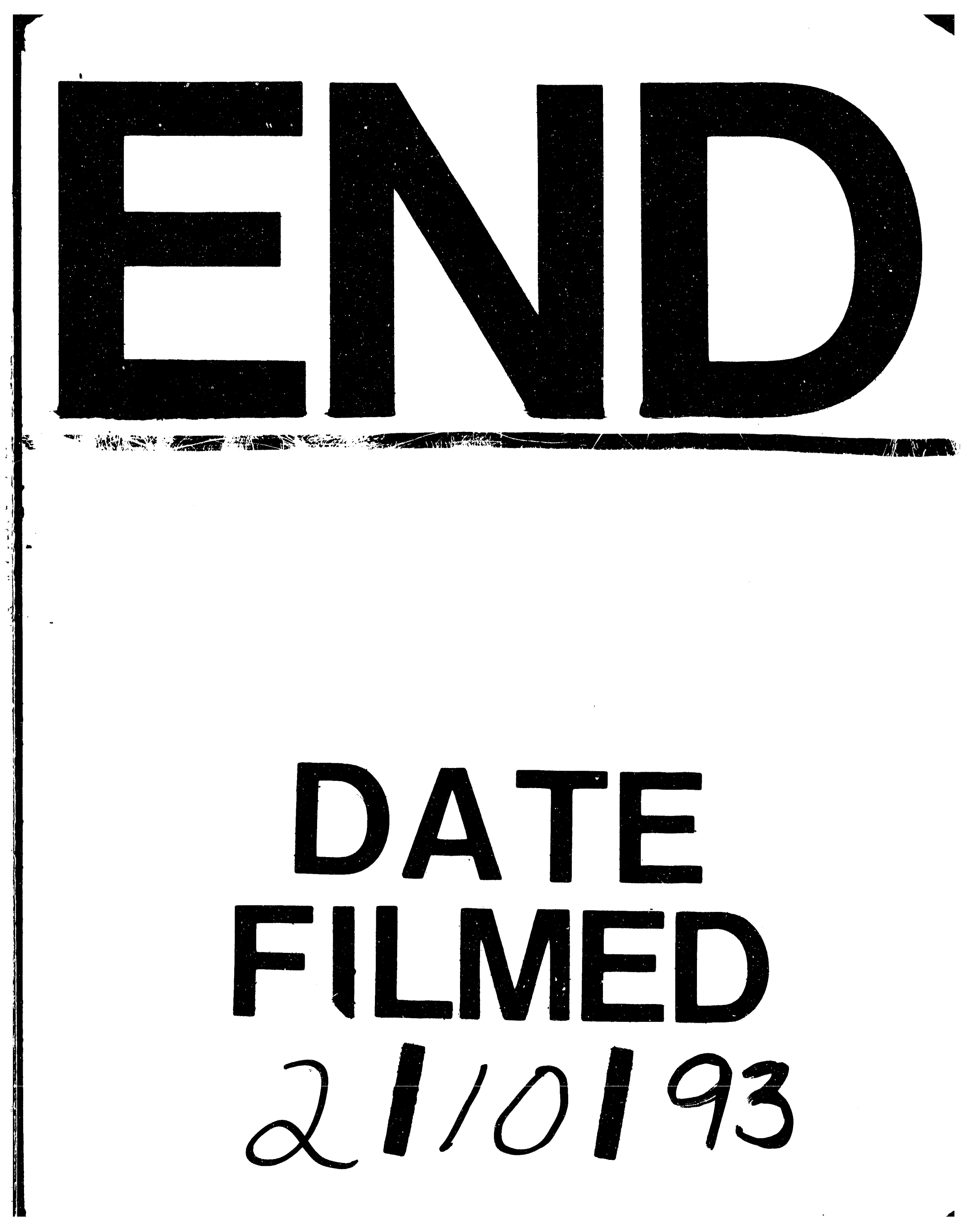




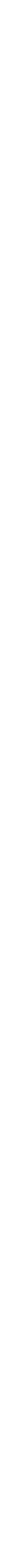

,
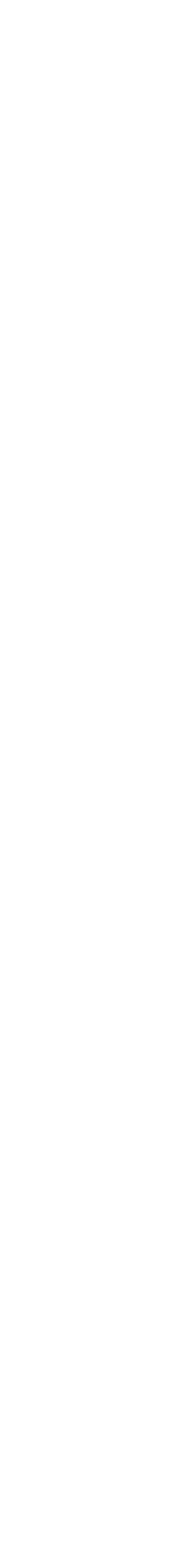